\title{
Novel Spectrophotometric Method for the Quantitation of Urinary Xanthurenic Acid and Its Application in Identifying Individuals with Hyperhomocysteinemia Associated with Vitamin $B_{6}$ Deficiency
}

\author{
Chi-Fen Chen, ${ }^{1}$ Tsan-Zon Liu, ${ }^{2}$ Wu-Hsiang Lan, ${ }^{1}$ Li-An Wu, ${ }^{3}$ Chin-Hung Tsai, ${ }^{4}$ \\ Jeng-Fong Chiou, ${ }^{5,6}$ and Li-Yu Tsai ${ }^{7}$ \\ ${ }^{1}$ Clinical Laboratories, Yuan's General Hospital, Kaohsiung 802, Taiwan \\ ${ }^{2}$ Translational Research Laboratory, Cancer Center, Taipei Medical University Hospital, Taipei 110, Taiwan \\ ${ }^{3}$ Clinical Laboratories, Chang Gung Memorial Hospital, Kwei-Shan 333, Taiwan \\ ${ }^{4}$ Department of Food Science, National Penghu University of Science and Technology, Magong, Penghu 880, Taiwan \\ ${ }^{5}$ Department of Radiation Oncology, School of Medicine, Taipei Medical University, Taipei 110, Taiwan \\ ${ }^{6}$ Cancer Center and Department of Radiation Oncology, Taipei Medical University and Hospital, Taipei 110, Taiwan \\ ${ }^{7}$ Division of Clinical Biochemistry, Graduate Institute of Medical Biotechnology, Kaohsiung Medical University, Kaohsiung 807, Taiwan
}

Correspondence should be addressed to

Jeng-Fong Chiou; sjfchiou@xuite.net and Li-Yu Tsai; tsailiyu2005@yahoo.com.tw

Received 20 June 2013; Accepted 16 August 2013

Academic Editor: Natasa Tosic

Copyright (c) 2013 Chi-Fen Chen et al. This is an open access article distributed under the Creative Commons Attribution License, which permits unrestricted use, distribution, and reproduction in any medium, provided the original work is properly cited.

\begin{abstract}
A novel spectrophotometric method for the quantification of urinary xanthurenic acid (XA) is described. The direct acid ferric reduction (DAFR) procedure was used to quantify XA after it was purified by a solid-phase extraction column. The linearity of proposed method extends from 2.5 to $100.0 \mathrm{mg} / \mathrm{L}$. The method is precise, yielding day-to-day CVs for two pooled controls of $3.5 \%$ and $4.6 \%$, respectively. Correlation studies with an established HPLC method and a fluorometric procedure showed correlation coefficients of 0.98 and 0.98 , respectively. Interference from various urinary metabolites was insignificant. In a smallscale screening of elderly conducted at Penghu county in Taiwan $(n=80)$, we were able to identify a group of twenty individuals having hyperhomocysteinemia ( $>15 \mu \mathrm{mole} / \mathrm{L}$ ). Three of them were found to be positive for XA as analyzed by the proposed method, which correlated excellently with the results of the activation coefficient method for RBC's AST/B $\mathrm{B}_{6}$ functional test. These data confirm the usefulness of the proposed method for identifying urinary XA as an indicator of vitamin $\mathrm{B}_{6}$ deficiency-associated hyperhomocysteinemic condition.
\end{abstract}

\section{Introduction}

Homocysteine (Hcy) is a sulfur-containing amino acid which is an intermediary product of the transmethylation reaction of methionine. Once formed, Hcy can either be remethylated back to methionine by a folate- and vitamin $\mathrm{B}_{12}$-dependent enzymatic reaction or can undergo the transsulfuration pathway to form cysteine (the rate-limiting precursor for gluthathione) via a two-step enzymatic process catalyzed by cystathionine $\beta$-synthase (CBS) and cystathionase, both requiring vitamin $\mathrm{B}_{6}$ coenzyme [1-3]. Park and Linkswiler [4] reported that urinary Hcy excretion increased considerably with six male volunteers who consumed a diet depleted of vitamin $B_{6}$ whereas several studies on experimental animals suggested that a vitamin $\mathrm{B}_{6}$ deficiency could result in Hcy accumulation [5-7]. Collectively, these reports implicate that measurement of Hcy is not a specific biomarker for deficiency of vitamin $\mathrm{B}_{6}$. In contrast, kynureninase (EC 3.7.1.3), a pyridoxal $5^{\prime}$-phosphate coenzyme-requiring enzyme is essential for the catalytic action of converting 3-hydroxykynurenin, 
an intermediary product of tryptophan metabolism, to 3hydroxyanthranilate [8]. Either subclinical deficiencies of vitamin $B_{6}$ or a genetic defeat on kynureninase can lead to the accumulation of xanthurenic acid (XA) in plasma and urine [5-7]. Collectively, despite vitamin $\mathrm{B}_{6}$ deficiency can lead to a combined accumulation of XA and Hcy in urine, the former is considered to be a more sensitive and specific indicator than $\mathrm{Hcy}$ for the evaluation of vitamin $\mathrm{B}_{6}$ deficiency because Hcy can be diverted to other pathways even in cases of vitamin $\mathrm{B}_{6}$ deficiency.

Vitamin $\mathrm{B}_{6}$ deficiency was first suggested earlier by scientists who demonstrated widespread vascular lesion in pyridoxine deficient monkeys with little or no lipid disposition, and their serum cholesterol were hardly elevated [9]. Furthermore, Ubbink et al. [10] reported that patients with cystathionine $\beta$-synthase (EC 4.2.1.22) deficiency, the first vitamin $\mathrm{B}_{6}$-dependent enzyme catalyzing the transsulfuration pathway of homocysteine catabolism, exhibited widespread vascular disorders. In addition, patients with rheumatoid arthritis had been reported to possess reduced circulating level of vitamin $\mathrm{B}_{6}$ and their plasma pyridoxal $5^{\prime}$ phosphate levels correlated with both the net Hcy increase in response to a methionine load test and $24 \mathrm{hr}$ urinary XA excretion in response to a tryptophan load test [11]. Besides, Zhang et al. [12] implicated that pyridoxal $5^{\prime}$-phosphate, the principal active form of vitamin $\mathrm{B}_{6}$, has a number of biological roles that potentially make it important in cancer. The rationale of this implication is that adequate vitamin $\mathrm{B}_{6}$ levels are important for conversion of Hcy into cysteine and high intracellular levels of pyridoxal $5^{\prime}$-phosphate can lead to decreased steroid hormone-induced gene expression. In addition, these authors also presented evidence that higher plasma levels of folate and vitamin $\mathrm{B}_{6}$ may reduce the risk of developing breast cancer. These data implies that vitamin $\mathrm{B}_{6}$ deficiency itself may be a risk factor for cancer.

Measurement of urinary or plasma XA has been used clinically to study vitamin $\mathrm{B}_{6}$ deficiency $[8,13,14]$, including febrile disorder [15], theophylline-induced asthma [16], druginduced diabetes [16], the effect of tryptophan and six of its metabolites on the nicotinic acid pathway [17], and the etiological role in a variety of chronic degenerative disease including a variety of cancers [18-22]. Among the methods developed for quantifying urinary XA [23-28], one tedious and lengthy assay involved the extraction of XA from urine with isobutanol, isolation by thin-layer chromatography, and eventually spectrophotometric determination [25]. The first fluorometric quantification of urinary XA was devised by Satoh and Price [23], based on the separation of XA by Dowex $50\left(\mathrm{H}^{+}\right)$, followed by measurement of its fluorescence in strong alkali, and kynurenic acid was simultaneously determined by fluorometry in strong $\mathrm{H}_{2} \mathrm{SO}_{4}$. This method was subsequently modified by Cohen et al. [24], who separated $\mathrm{XA}$ from other fluorescent substances in urine by a $\mathrm{pH}-$ and $\mathrm{NaCl}$-dependent extraction with isobutanol and then determined the fluorescence of XA in alkaline solution so that potentially interfering compounds such as kynurenic acid could be obviated. However, all these methods involve multiple procedural steps which are rather tedious and timeconsuming. In addition, several HPLC methods have also been devised for quantifying urinary XA $[26,28]$. However, the expensive instruments required might not be readily available in a general clinical laboratory. For these reasons, we set out to develop this rapid and simple spectrophotometric method for measuring urinary XA that is free of interference and can be easily adoptable for routine use by clinical and micronutrient assessment laboratories. To further exemplify its clinical application, we performed a single-blind study on a group of urine specimens $(N=20)$ obtained from patients with hyperhomocysteinemia of unknown etiologies using proposed method to screen for XA. Based on this screening procedure, we were able to successfully identify three out of twenty patients with hyperhomocysteinemia that were actually originated from vitamin $\mathrm{B}_{6}$ deficiency.

\section{Material and Methods}

2.1. Chemicals. Unless otherwise stated, reagents of the highest quality available were obtained commercially. XA (4,8-dihydroxyquinaldic acid), kynurenic acid (4-hydroxyquinoline-2-carboxylic acid), 2,4,6-tris-(pyridyl)-s-triazine (TPTZ), ferric chloride hexahydrate, vitamin C, uric acid, salicylate, acetaminophen, vanillylmandelic acid, and homovanillic acid were purchased from Sigma Chemical Co. (St. Louis, MO, USA). Solid-phase anionic-exchange resin (trimethylaminopropyl group bound to silica) was purchased from Analytichem (Harbor City, CA).

2.2. Reagents. (a) Acetate buffer, $0.3 \mathrm{~mol} / \mathrm{liter}, \mathrm{pH} 3.6$, is prepared by dissolving $3.1 \mathrm{~g}$ of sodium acetate trihydrate in distilled water. Add $16.0 \mathrm{~mL}$ of glacial acetic acid. Dilute to 1 liter, check the $\mathrm{pH}$, and adjust it to 3.6. (b) TPTZ, $8 \mathrm{mmol} /$ liter, is prepared by dissolving $624 \mathrm{mg}$ of TPTZ in $250 \mathrm{~mL}$ of $\mathrm{HCl}$ solution $(36 \mathrm{mmol} /$ liter, $0.75 \mathrm{~mL}$ conc. $\mathrm{HCl}$ and $\mathrm{H}_{2} \mathrm{O}$ ). (c) $\mathrm{FeCl}_{3} \cdot 6 \mathrm{H}_{2} \mathrm{O}$ solution is prepared by dissolving $540 \mathrm{mg}$ of ferric chloride hexahydrate in $100 \mathrm{~mL} \mathrm{HCl}$ solution ( $0.02 \mathrm{~mol} /$ liter, $0.16 \mathrm{~mL}$ of conc. $\mathrm{HCl}$, and $\mathrm{H}_{2} \mathrm{O}$ ).

2.3. Urine Purification. Routinely, 24 h urine specimens are collected in brown bottles with $6 \mathrm{~N} \mathrm{HCl}$ as preservative and proceed for analyses without delay. The sample should be well mixed and an aliquot frozen if the sample cannot be analyzed within $48 \mathrm{hr}$ after collection. Before analysis, the urine specimen (after thawing, if necessary) is filtered through Whatman No. 1 filter paper, and $5.0 \mathrm{~mL}$ of urine is applied to an anion-exchange solid-phase extraction column (100 mg/column). After the urine sample has passed through the extraction column, the adsorbed XA is eluted with $2.0 \mathrm{~mL}$ of $0.1 \mathrm{M} \mathrm{HCl}$.

2.4. Spectrophotometry of XA. $0.5 \mathrm{~mL}$ of eluate from purified procedure mentioned previously is added and $0.5 \mathrm{~mL}$ of $1 \mathrm{~N} \mathrm{NaOH}$. $0.1 \mathrm{~mL}$ of this alkaline elute is then added to a solution containing $2.5 \mathrm{~mL}$ of acetate buffer, $\mathrm{pH} 3.6,0.3 \mathrm{~mL}$ of TPTZ, and $0.1 \mathrm{~mL}$ of $\mathrm{FeCl}_{3}$ solution. The tubes are well mixed and stand at room temperature for exactly 15 minutes. Immediately, the absorbance of each tube is read at $593 \mathrm{~nm}$. 
XA concentration of the unknown is estimated from the standard curve based on the absorbances of the standards.

2.5. Fluorometric Measurement of $X A$. The eluate containing $\mathrm{XA}$ from urine is dissolved into potassium phosphate buffer, $\mathrm{pH}$ 4.0. The fluorescence of XA is then determined at 460$470 \mathrm{~nm}$ (after excitation at $305 \mathrm{~nm}$ ) with a Turner spectrofluorometer.

2.6. Correlation Studies. The results obtained by the proposed method were compared with those determined by an established HPLC method [27] and with a fluorometric method established in our laboratory [29].

2.7. Rapid Screening of Urinary XA in Elderly with Hyperhomocysteinemic Condition. We conducted a small-scale screening of the elderly (age 61 to 80 years) $(n=80)$ for hyperhomocysteinemia during a regular annual physical check up from Penghu County. The project was approved by the Institutional Review Board of Kaohsiung Medical University (KMUH-IRB-960036). Individuals who had hyperhomocysteinemic condition $(>15 \mu \mathrm{mole} / \mathrm{L})$ were asked by the physician to donate urine specimens with informed consent. These specimens were then analyzed by our proposed method for XA. For those urine samples positive for $\mathrm{XA}$, a parallel determination of activation coefficients on RBC's aspartic acid aminotransferase (AST)/B-6 functional test was performed to confirm if XA-positive individuals were definitively deficient in vitamin $\mathrm{B}_{6}$.

2.8. Statistics. Data are expressed as mean \pm S.D. and analyzed using Student's $t$-test. $P$ values lower than 0.05 were considered statistically significant.

\section{Results}

3.1. Absorption Spectra. Spectral scan for XA after reacting with $\mathrm{Fe}^{+3}$-TPTZ complex in acidic buffer ( $\mathrm{pH}$ 3.6) exhibit a maximum absorption peak at $593 \mathrm{~nm}$. The absorption peak represents the formation of $\mathrm{Fe}^{+2}$-TPTZ (intense blue color) (Figure 1).

3.2. Time Course Study. Reduction of $\mathrm{Fe}^{+3}$-TPTZ complex by XA exhibits a time-dependent manner. The increment of absorption at $593 \mathrm{~nm}$ reaches a plateau approximately $30 \mathrm{~min}$ (Figure 2). For accuracy, the reaction time for the proposed method should be set at $30 \mathrm{~min}$. If this procedural step is inconvenient, a short time of color development can be used (e.g., $15 \mathrm{~min}$ ), but each tube should be timed for equal amount of incubation time, and the absorbance at $593 \mathrm{~nm}$ should be read immediately without delayed.

3.3. Linearity. Absorbances of calibrators at $593 \mathrm{~nm}$ were linearly related to XA concentrations from 2.5 to $100 \mathrm{mg} / \mathrm{L}$ (Figure 3). The limit of detection (LOD) is $10 \mu \mathrm{g} / \mathrm{mL}$.

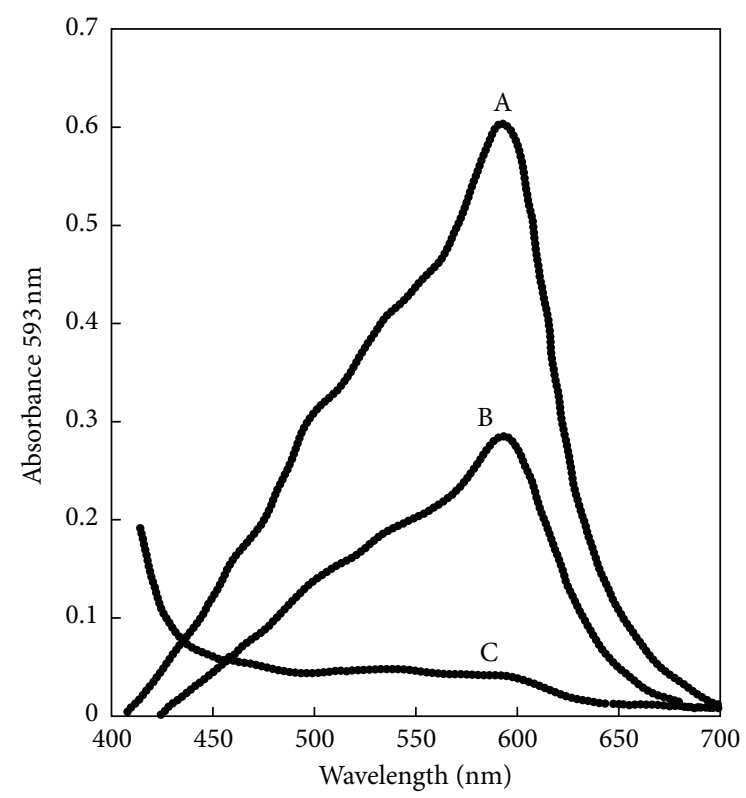

FIGURE 1: Spectral scan for xanthurenic acid after reacting with $\mathrm{Fe}^{3+}$. TPTZ complex in acidic buffer $(\mathrm{pH}=3.6)$ exhibiting a maximum absorption peak at $593 \mathrm{~nm}$. The absorption peak represents the formation of $\mathrm{Fe}^{2+}$-TPTZ (intense blue color). $\mathrm{A}=8 \mu \mathrm{g} \mathrm{XA}$ in $0.1 \mathrm{~mL}$ $\mathrm{MeOH}$ versus reagent blank, $\mathrm{B}=4 \mu \mathrm{g} \mathrm{XA}$ in $0.1 \mathrm{~mL} \mathrm{MeOH}$ versus reagent blank, and $\mathrm{C}=$ reagent blank versus $\mathrm{H}_{2} \mathrm{O}$.

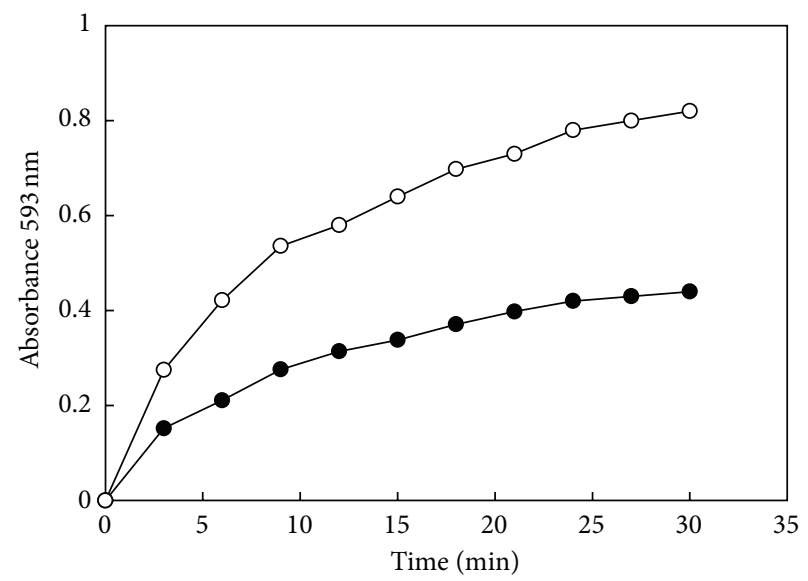

FIGURE 2: Reduction of $\mathrm{Fe}^{3+}-\mathrm{TPTZ}$ complex by XA is a timedependent process. The increment of absorption at $593 \mathrm{~nm}$ reaches a plateau at approximately $30 \mathrm{~min}$. For accuracy, the reaction time for the proposed method should be set at $30 \mathrm{~min}$. $(\bullet-\bullet)=4 \mu \mathrm{g} \mathrm{XA}$ in $\mathrm{MeOH}$ and $(\mathrm{O}-\mathrm{O})=8 \mu \mathrm{g}$ XA in $\mathrm{MeOH}$.

3.4. Analytical Recovery. To determine the accuracy of the procedure, we performed a recovery study the percentage of recovery represents the measured value expressed as a percentage of the expected value. The mean percentage of recovery for 10 samples was $99.8 \pm 2.1 \%$ (data not shown).

3.5. Precision. Reproducibility as reflected by day-to-day and within-run precision data were excellent (Table 1). Five repetitive determinations on two pooled XA-supplemented 


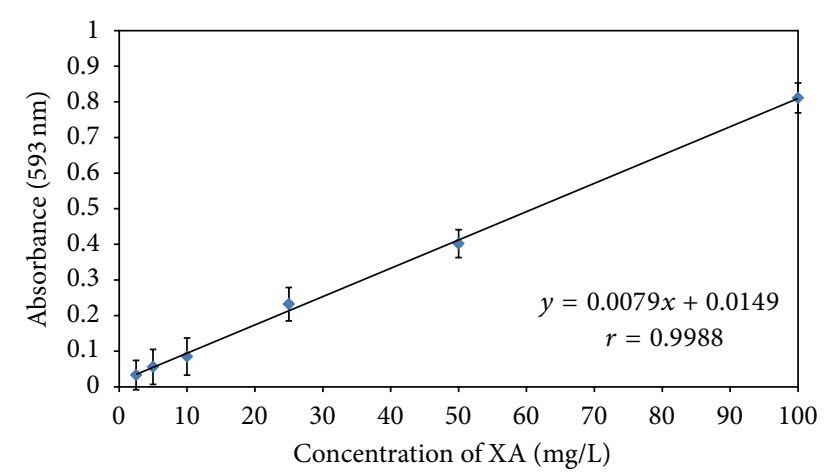

FIGURE 3: Linearity of XA determination as assayed by the proposed spectrophotometric method. Each point represents an average of triplicate determinations. The XA stock solution $(100 \mathrm{mg} / \mathrm{L})$ was prepared by dissolving $10 \mathrm{mg}$ of XA into $1.0 \mathrm{~mL}$ of methanol and then made up to $100 \mathrm{~mL}$ with XA-free pooled urine. Various concentrations of standards were then made up by diluting with XAfree urine to the desired concentrations.

TABLE 1: Precision studies results.

\begin{tabular}{cc}
$\begin{array}{c}\text { XA determined by } \\
\text { the proposed } \\
\text { method }(\mathrm{mg} / \mathrm{L})^{*}\end{array}$ & $\begin{array}{c}\text { Indirect activation } \\
\text { coefficient }(\mathrm{AC}) \text { test } \\
\text { for RBC/AST }\end{array}$ \\
\hline
\end{tabular}

\begin{tabular}{lcc}
\hline Within-run $(n=5)^{*}$ & & \\
Mean, mg/L & 20.2 & 41.0 \\
SD, mg/L & 0.60 & 1.5 \\
CV, \% & 3.0 & 3.7 \\
Day-to-day $(n=25)^{* *}$ & & \\
Mean, mg/L & 20.2 & 41.0 \\
SD, mg/L & 0.7 & 1.9 \\
CV, \% & 3.5 & 4.6 \\
\hline
\end{tabular}

${ }^{*}$ Each level of pooled urine sample was determined simultaneously for 5 times at the same day.

${ }^{* *}$ Each level of pooled urine sample was run 5 times per day for five consecutive days. The pooled data were then calculated for mean, SD and CV.

urine controls had a mean value of 20.2 and $41.0 \mathrm{mg} / \mathrm{L}$, respectively (with CVs of $<5.0 \%$ ). The CVs for the same set of controls assayed on 5 consecutive days were $3.5 \%$ and $4.6 \%$, respectively. Again, the day-to-day reproducibility was also excellent.

3.6. Correlation Studies. We compared results by the proposed method with those determined by an established HPLC method [27] for 30 samples. The correlation coefficient between the two methods was 0.98. (Figure 4(a)) Comparison of the proposed method with a fluorometric method [29] for 30 samples showed that the correlation coefficient between the two methods was also 0.98 (Figure 4(b)).

3.7. Interference Assay. The purification step with the solidphase anion-exchange column presumably renders the assay free of interferences. However, to verify this assumption, we checked for possible interference from the following commonly encountered substances in urine: vitamin C, uric acid,
TABLE 2: A single blind study for 20 hyperhomocysteinemic urine specimens obtained through a small-scale screening of the elderly $(n=80)$ for assaying XA by our proposed method and their parallel comparison of assessing $\mathrm{B}_{6}$ status by indirect activation coefficient test for RBC/AST.

\begin{tabular}{lcc}
\hline $\begin{array}{l}\text { Specimen } \\
\text { code no. }\end{array}$ & $\begin{array}{c}\text { XA determined by the } \\
\text { proposed method } \\
(\mathrm{mg} / \mathrm{L})\end{array}$ & $\begin{array}{c}\text { Indirect activation } \\
\text { coefficient (AC) test for } \\
\text { RBC/AST }\end{array}$ \\
\hline 1 & ND & Normal \\
2 & ND & Normal \\
3 & ND & Normal \\
4 & 13.5 & Abnormal $(\mathrm{AC}=1.51)$ \\
5 & ND & Normal \\
6 & ND & Normal \\
7 & 14.2 & Abnormal (AC $=1.43)$ \\
8 & ND & Normal \\
9 & ND & Normal \\
10 & ND & Normal \\
11 & ND & Normal \\
12 & ND & Abnormal (AC $=1.80)$ \\
13 & 25.3 & Normal \\
14 & ND & Normal \\
15 & ND & Normal \\
16 & ND & Normal \\
17 & ND & Normal \\
18 & ND & Normal \\
19 & ND & Normal \\
${ }^{* *}$ AD $:$ nondetectable $(<$ LOD $)$. & is designated as normal. & \\
& &
\end{tabular}

salicylate, vanillylmandelic acid (VMA), and homovanillic (HVA). At concentrations of $0.5 \mathrm{~g} / \mathrm{L}$, all of these compounds gave no interference with the assay.

\section{Discussion}

The described method for the measurement of XA in urine was evaluated for its specificity, accuracy, and reproducibility for general use. First, the solid-phase extraction column (trimethylaminopropyl group bound to silica) concentrates not only the desired XA, but also leave behind urinary substances such as the tryptophan metabolites, kynurenin, and hydroxylkynurenine. This purification step for XA confers a unique specificity to the proposed method.

During the course of this developmental work, we considered incorporating a hydrolytic step to account for the possible presence of conjugated forms of XA. Rothstein and Greenberg [30] have previously reported that the urinary XA is conjugated as glucuronide in the rat and as the sulfate in the rabbit. In contrast, Wallace et al. [25] showed that the amount of XA in human urine remained steady after hydrolysis with acid or glucuronidase, indicating that very little conjugated XA was present. This observation was also 


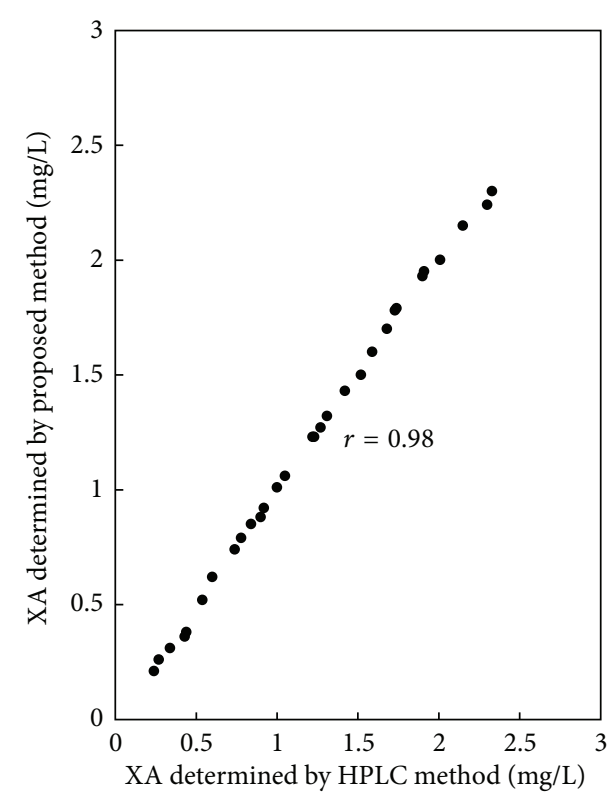

(a)

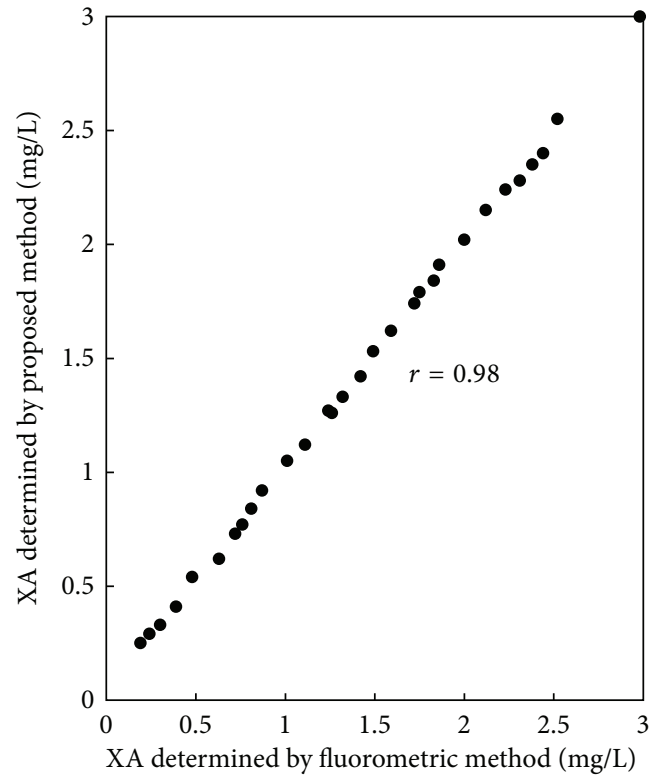

(b)

FIGURE 4: Correlation of results of XA concentrations obtained by the proposed method and those determined by an established HPLC method (a) or with an established fluorometric procedure (b).

previously confirmed by us [29]. Thus, we decided not to incorporate a hydrolysis step for urine in our proposed method.

The accuracy of the method, evaluated by measuring XA added to pooled urine in which no endogenous XA was detected that shows the mean percentage of recovery for 10 samples was $99.8 \pm 2.1 \%$ (date not shown). Additionally, reproducibility as reflected by day-to-day and within-run precision data was also excellent (Table 1).

It has been well documented that one of the etiologic factors underlying chronic degenerative diseases such as cancer, diabetes, and Alzheimer's diseases may be oxidative stress to which the elderly population is specially prone. In addition, the elderly population may have deficiencies of several micronutrients including folate, pyridoxine, and cobalamin; if not treated in time, these deficiencies can lead to an accumulation of both XA and Hcy in the urine or plasma and present a serious health risk to these individuals. As an example, Wilson et al. [31] reported that about $10 \%$ of the US population consumes less than half of the RDA ( $1.6 \mathrm{mg} /$ day) of vitamin $B_{6}$. As also pointed out by Ames [32], vitamin $\mathrm{B}_{6}$ deficiency can cause a decrease in enzyme activity of serine hydroxylmethyltransferase which supplies the methylene group for methylene-THF [33]. If the methylene-THF pool is decreased in $\mathrm{B}_{6}$ deficiency, an episode of uracil incorporation into DNA can be anticipated to cause chromosome breaks and the risk for cancer will be greatly increased. In a casecontrol study of diet and cancer, vitamin $\mathrm{B}_{6}$ intake was inversely associated with prostate cancer [34]. Thus, we think that it is of importance to undertake a large-scale screening to identify individuals from an elderly population who maybe deficient in these micronutrients. Our proposed method is sample and rapid and can be modified to suit for semiautomated quantification of vitamin $\mathrm{B}_{6}$ deficiency in a large scale screening purpose. More importantly, it can also be adopted as one of the tests for routine use in the clinical laboratory. Our small scale screening for elderly who were hyperhomocysteinemic followed by correctly identified individuals high in urinary $\mathrm{XA}$ which was indicative of vitamin $\mathrm{B}_{6}$ deficiency can serve as a testimony of the applicability of our proposed method (Table 2). Further study is urgently needed to establish if there is a correlation between vitamin $\mathrm{B}_{6}$ deficiency-induced hypercysteinemia and the risk of cancer. Our proposed noninvasive urinary XA test is well suited for the investigation of this kind.

\section{Conclusion}

This work describes a simple and rapid spectrophotometric method for quantifying urinary XA as an indicator of vitamin $\mathrm{B}_{6}$ deficiency which allows one to differentiate hyperhomocysteinemic condition independent of folate/vitamin B12 involvement. We also exemplifiy its application by correctly identified these individuals whose hyperhomocysteinemia condition were solely derived from vitamin $\mathrm{B}_{6}$ deficiency. This noninvasive spectrophotometric method for quantifying urinary XA may also be suitable for identifying individuals with vitamin $B_{6}$ deficiency and subsequently receiving vitamin $B_{6}$ supplementation to reduce the risk of cancer due to Hcy-evoked oxidative stress.

\section{Conflict of Interests}

The authors declare they have no conflict of interests. 


\section{Authors' Contribution}

J.-F. Chiou and L.-Y. Tsai are cocorresponding authors.

\section{Acknowledgments}

The expert technical assistance of Ms. S. Y. Mau is greatly appreciated.

\section{References}

[1] J. D. Finkelstein, "Methionine metabolism in mammals," Journal of Nutritional Biochemistry, vol. 1, no. 5, pp. 228-237, 1990.

[2] S. C. Lu and J. M. Mato, "S-adenosylmethionine in cell growth, apoptosis and liver cancer," Journal of Gastroenterology and Hepatology, vol. 23, supplement 1, pp. S73-S77, 2008.

[3] O. Midttun, S. Hustad, and P. M. Ueland, "Quantitative profiling of biomarkers related to B-vitamin status, tryptophan metabolism and inflammation in human plasma by liquid chromatography/tandem mass spectrometry," Rapid Communications in Mass Spectrometry, vol. 23, no. 9, pp. 1371-1379, 2009.

[4] Y. K. Park and H. Linkswiler, "Effect of vitamin B6 depletion in adult man on the excretion of cystathionine and other methionine metabolites," Journal of Nutrition, vol. 100, no. 1, pp. 110-116, 1970.

[5] M. Slavik, K. J. Smith, and O. Blanc, "Decrease of serum pyridoxal phosphate levels and homocystinemia after administration of 6-azauridine triacetate and their prevention by administration of pyridoxine," Biochemical Pharmacology, vol. 31, no. 24, pp. 4089-4092, 1982.

[6] L. A. Smolin and N. J. Benevenga, "Accumulation of homocyst(e)ine in vitamin B-6 deficiency: a model for the study of cystathionine $\beta$-synthase deficiency," Journal of Nutrition, vol. 112, no. 7, pp. 1264-1272, 1982.

[7] L. A. Smolin, T. D. Crenshaw, D. Kurtycz, and N. J. Benevenga, "Homocyst(e)ine accumulation in pigs fed diets deficient in vitamin B-6: relationship to atherosclerosis," Journal of Nutrition, vol. 113, no. 10, pp. 2022-2033, 1983.

[8] F. Takeuchi, R. Tsubouchi, S. Izuta, and Y. Shibata, "Kynurenic metabolism and xanthurenic acid formation in vitamin B6deficient rat after tryptophan injection," Journal of Nutritional Science and Vitaminology, vol. 35, no. 2, pp. 111-122, 1989.

[9] S. J. Chang, "Vitamin B6 antagonists alter the function and ultrastructure of mice endothelial cells," Journal of Nutritional Science and Vitaminology, vol. 46, no. 4, pp. 149-153, 2000.

[10] J. B. Ubbink, W. J. H. Vermaak, A. van der Merwe, and P. J. Becker, "Vitamin B-12, vitamin B-6, and folate nutritional status in men with hyperhomocysteinemia," The American Journal of Clinical Nutrition, vol. 57, no. 1, pp. 47-53, 1993.

[11] E. P. I. Chiang, J. Selhub, P. J. Bagley, G. Dallal, and R. Roubenoff, "Pyridoxine supplementation corrects vitamin B6 deficiency but does not improve inflammation in patients with rheumatoid arthritis," Arthritis Research \& Therapy, vol. 7, no. 6, pp. R1404R1411, 2005.

[12] S. M. Zhang, W. C. Willett, J. Selhub et al., "Plasma folate, vitamin B6, vitamin B12, homocysteine, and risk of breast cancer," Journal of the National Cancer Institute, vol. 95, no. 5, pp. 373-380, 2003.

[13] D. A. Bender, E. N. M. Njagi, and P. S. Danielian, "Tryptophan metabolism in vitamin B6-deficient mice," The British Journal of Nutrition, vol. 63, no. 1, pp. 27-36, 1990.
[14] J. E. Leklem, “Vitamin B-6: a status report," Journal of Nutrition, vol. 120, no. 11, pp. 1503-1507, 1990.

[15] R. C. Shaw and R. D. Feigin, "Excretion of kynurenic acid and xanthurenic acid during infection," Pediatrics, vol. 47, no. 1, pp. 47-56, 1971.

[16] J. B. Ubbink, R. Delport, P. J. Becker, and S. Bissbort, "Evidence of a theophylline-induced vitamin B6 deficiency caused by noncompetitive inhibition of pyridoxal kinase," Journal of Laboratory and Clinical Medicine, vol. 113, no. 1, pp. 15-22, 1989.

[17] S. Ikeda and Y. Kotake, "Urinary excretion of xanthurenic acid and zinc in diabetes: (3). Occurrence of xanthurenic acid- $\mathrm{Zn}^{2+}$ complex in urine of diabetic patients and of experimentallydiabetic rats," Italian Journal of Biochemistry, vol. 35, no. 4, pp. 232-241, 1986.

[18] J. V. Woodside, D. G. Fogarty, J. H. Lightbody et al., "Homocysteine and B-group vitamins in renal transplant patients," Clinica Chimica Acta, vol. 282, no. 1-2, pp. 157-166, 1999.

[19] E. P. I. Chiang, P. J. Bagley, R. Roubenoff, M. Nadeau, and J. Selhub, "Plasma pyridoxal $5^{\prime}$-phosphate concentration is correlated with functional vitamin B-6 indices in patients with rheumatoid arthritis and marginal vitamin B-6 status," Journal of Nutrition, vol. 133, no. 4, pp. 1056-1059, 2003.

[20] C. C. Lin and M. C. Yin, "B vitamins deficiency and decreased anti-oxidative state in patients with liver cancer," European Journal of Nutrition, vol. 46, no. 5, pp. 293-299, 2007.

[21] E. Schernhammer, B. Wolpin, N. Rifai et al., "Plasma folate, vitamin $\mathrm{B} 6$, vitamin $\mathrm{B} 12$, and homocysteine and pancreatic cancer risk in four large cohorts," Cancer Research, vol. 67, no. 11, pp. 5553-5560, 2007.

[22] E. Ma, M. Iwasaki, I. Junko et al., "Dietary intake of folate, vitamin B6, and vitamin B12, genetic polymorphism of related enzymes, and risk of breast cancer: a case-control study in Brazilian women," BMC Cancer, vol. 9, article 122, 2009.

[23] K. Satoh and J. M. Price, "Fluorometric determination of kynurenic acid and xanthurenic acid in human urine," The Journal of Biological Chemistry, vol. 230, no. 2, pp. 781-789, 1958.

[24] G. Cohen, R. A. Fishman, and A. L. Jenkins, "A fluorometric method for the determination of xanthurenic acid in urine," Journal of Laboratory and Clinical Medicine, vol. 67, no. 3, pp. 520-527, 1966.

[25] M. J. Wallace, H. W. Vaillant, and H. A. Salhanick, "Method for quantitative measurement of xanthurenic acid in urine," Clinical Chemistry, vol. 17, no. 6, pp. 505-511, 1971.

[26] S. A. Williams, J. A. Monti, L. R. Boots, and P. E. Cornwell, "Quantitation of xanthurenic acid in rabbit serum using high performance liquid chromatography," The American Journal of Clinical Nutrition, vol. 40, no. 1, pp. 159-167, 1984.

[27] J. B. Ubbink, A. M. Schnell, and C. H. Rapley, "Quantification of urinary xanthurenic acid excretion by anion-exchange solidphase extraction and high-performance liquid chromatography," Journal of Chromatography, vol. 425, no. 1, pp. 182-186, 1988.

[28] E. Ohtsuki, M. Suzuki, M. Takayanagi, and T. Yashiro, "Colorimetric determination of urinary xanthurenic acid using an oxidative coupling reaction with N,N-diethyl-pphenylenediamine," Biological and Pharmaceutical Bulletin, vol. 17, no. 1, pp. 139-141, 1994.

[29] M. Liu, G. R. Wang, T. Z. Liu, and K. J. Tsai, "Improved fluorometric quantification of urinary xanthurenic acid," Clinical Chemistry, vol. 42, no. 3, pp. 397-401, 1996. 
[30] M. Rothstein and D. M. Greenberg, "Studies on the metabolism of xanthurenic acid-4-C14," Archives of Biochemistry and Biophysics, vol. 68, no. 1, pp. 206-214, 1957.

[31] J. W. Wilson, C. W. Enns, J. D. Goldman et al., "Data table: combined results from USDA's 1994 and 1995 continuing survey of food intakes by individuels and 1994 and 1995 diet and health knowledge survey," USDA/ARS Food Surveys Research Group, Beltsville Human Nutrition Research Center, Riverdale, Md, USA, 1997.

[32] B. N. Ames, "Micronutrient deficiencies cause DNA damage and cancer," Food Science and Agricultural Chemistry, vol. 1, pp. $1-15,1999$.

[33] S. P. Stabler, D. A. Sampson, L. P. Wang, and R. H. Allen, "Elevations of serum cystathionine and total homocysteine in pyridoxine-, folate-, and cobalamin-deficient rats," Journal of Nutritional Biochemistry, vol. 8, no. 5, pp. 279-289, 1997.

[34] T. J. Key, P. B. Silcocks, G. K. Davey, P. N. Appleby, and D. T. Bishop, "A case-control study of diet and prostate cancer," The British Journal of Cancer, vol. 76, no. 5, pp. 678-687, 1997. 

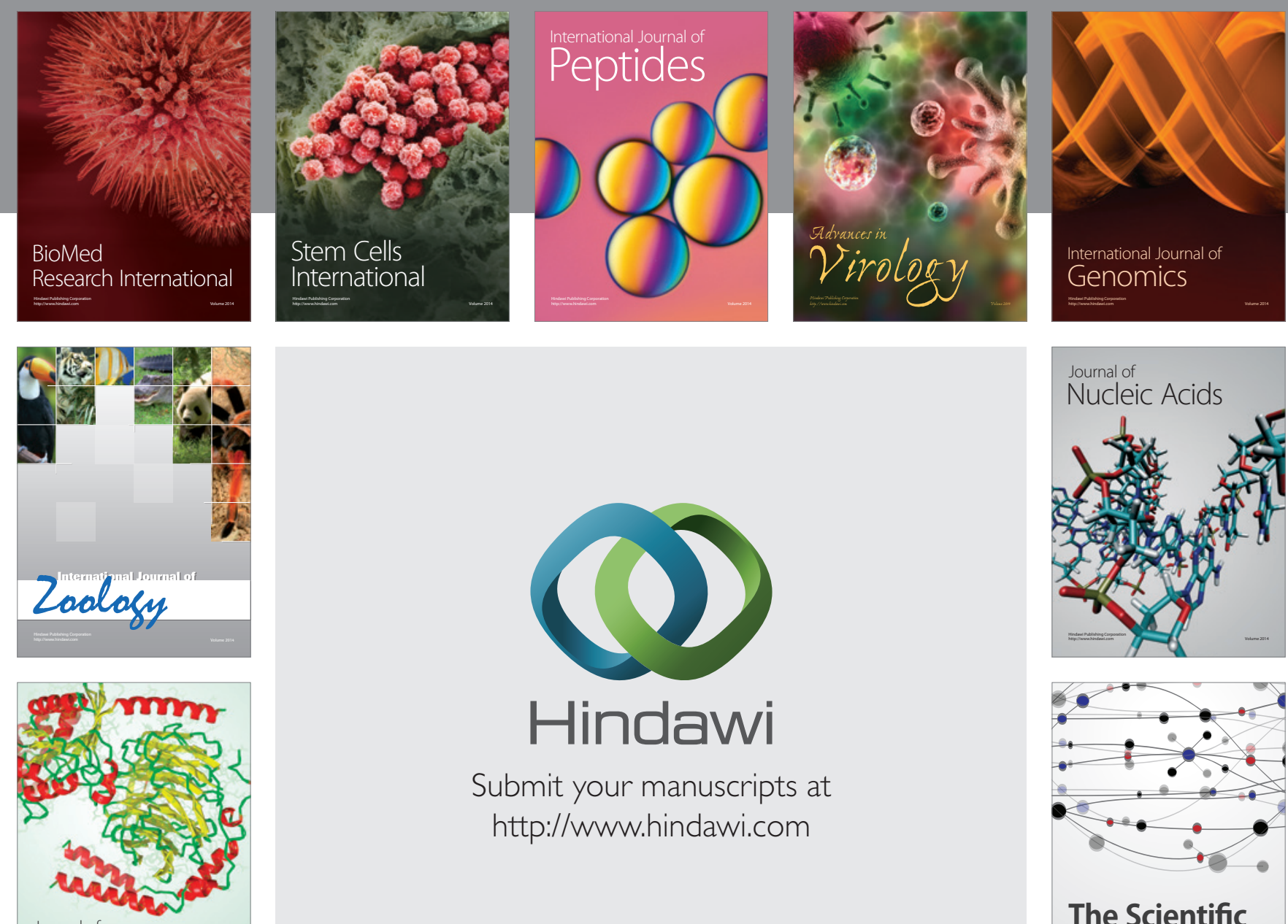

Submit your manuscripts at

http://www.hindawi.com

Journal of
Signal Transduction
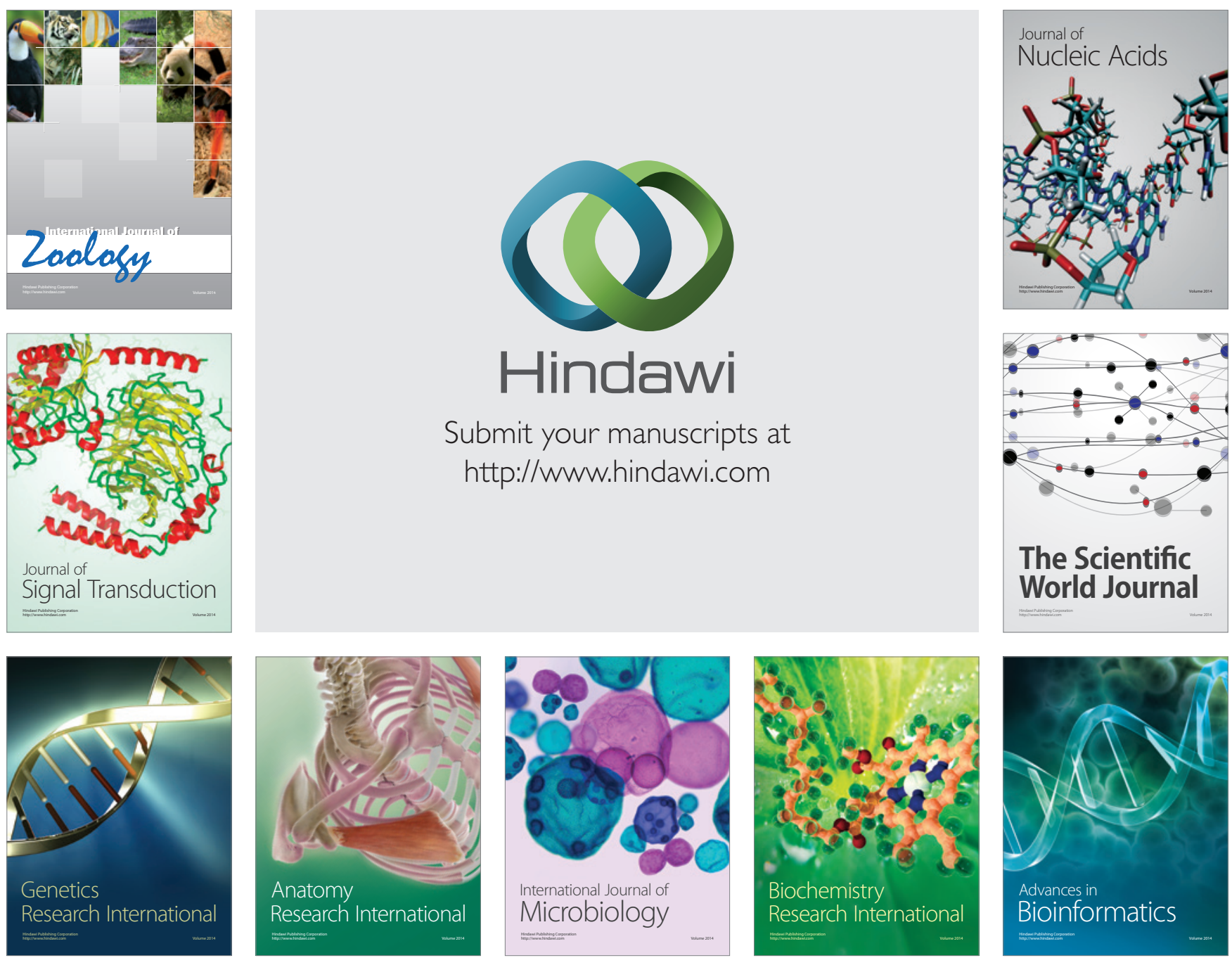

The Scientific World Journal
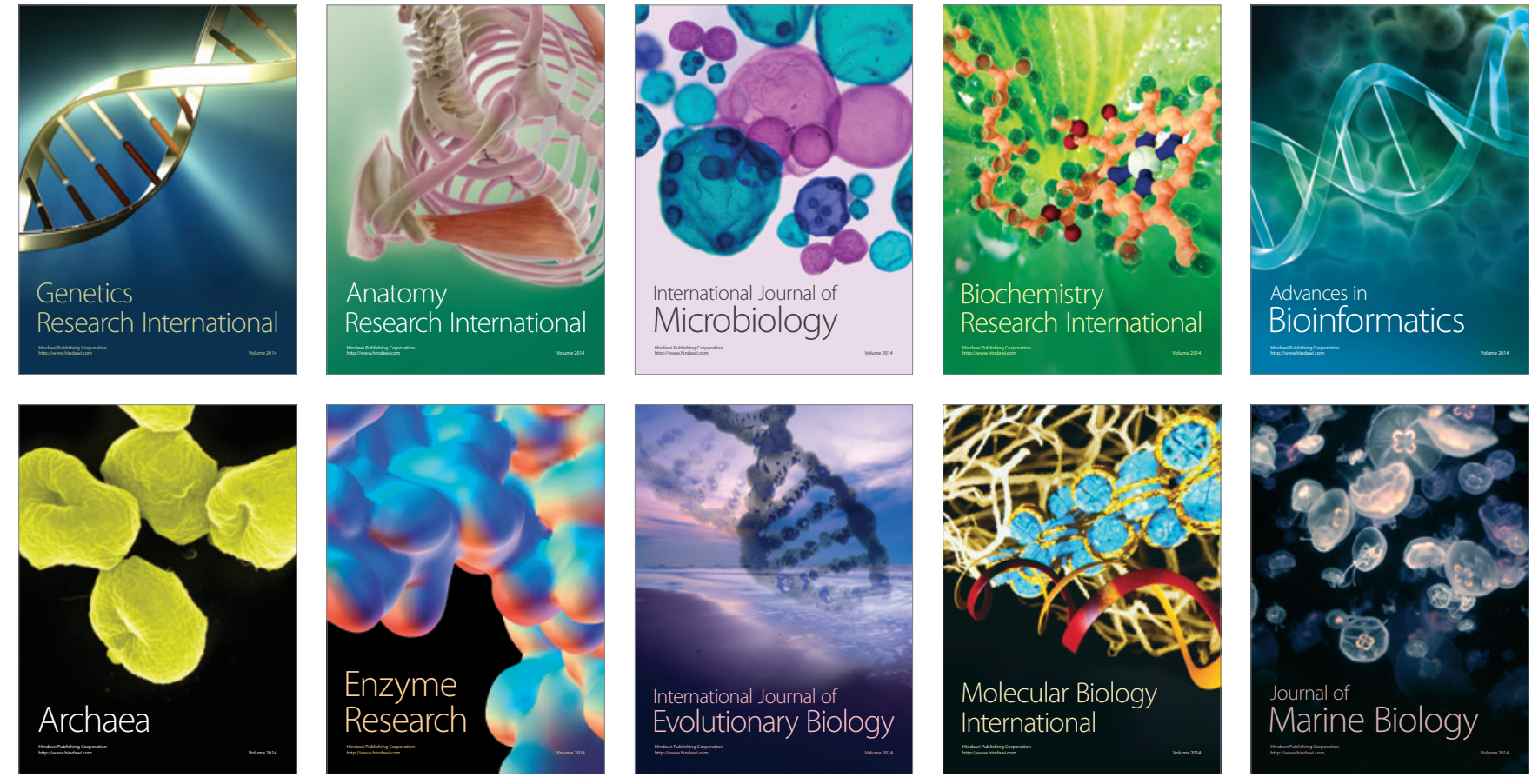